\title{
Realization of Low-Cost Multichannel Surface Plasmon Resonance Based Optical Transducer
}

\author{
Manjunath SOMARAPALLI*, Romuald JOLIVOT, and Waleed MOHAMMED \\ Bangkok University Centre of Research in Optoelectronics, Communications and Computational Systems, Bangkok \\ University, Bangkok 12120, Thailand \\ *Corresponding author: Manjunath SOMARAPALLIＩE-mail: s.manjunath005@gmail.com
}

\begin{abstract}
This paper demonstrates a low-cost and portable multichannel surface plasmon resonance (SPR) based optical transducer. The system's portability is achieved through the development of compact web-cam based spectrometer, and edge coupling to the SPR chip. Here, two configurations are presented: single-channel integrated system and two-channel system where the SPR chip and the spectrometer are coupled by a pair of plastic optical fibers. For the two-channel configuration, two different approaches are utilized to extract the optical spectrum: manual region cropping and automatic regions detection. For both approaches, image distortion and the size of the fiber tip affect the measured spectrum. For all configurations, mechanical alignment and mounting are made by 3D printing. The developed systems are tested with water and glycerol solution of different concentrations. The measured sensitivity is in the order of $10^{-4}$ RIU (refractive index unit) for all systems under the ambient condition.
\end{abstract}

Keywords: Plasmon; optical diffraction grating; optical fibers; spectrometer; 3D design; image processing

Citation: Manjunath SOMARAPALLI, Romuald JOLIVOT, and Waleed MOHAMMED, "Realization of Low-Cost Multichannel Surface Plasmon Resonance Based Optical Transducer," Photonic Sensors, Photonic Sensors, 2018, 8(4): $289-302$.

\section{Introduction}

Surface plasmon resonance (SPR) based optical transducer has been used as an optical sensing tool for many years. The high sensitivity of the resonance condition to the change in the surrounding refractive index (RI) has enabled SPR systems to be used in various applications such as detection of harmful gases in environment [1], determination of antibiotics in water [2], detection of protein binding [3], detection of heavy metal ions in water [4], and detection of hepatitis B (as biosensor) [5]. The development of low-cost and portable SPR systems for sensing applications has been demonstrated by many researchers based on the Kretschmann configuration [4-6], Otto configuration [7], diffractive optical coupling (DOC) [8], edge coupling for optical fibers [9], and also edge coupling to SPR chips in BU-SPR configuration as it is proposed earlier by the authors [10]. The SPR signal detection can be done through intensity and angular modulation technique, which employs a photo detectors (with moving parts) or webcam for the SPR detection [11, 12]. The wavelength and angular modulation techniques utilize spectrometer or charge coupled device (CCD) array [13]. Very few systems have demonstrated a low-cost SPR system along with signal detection. One of them is SPREETA [14], in which the light source, SPR chip, mirror, and a CCD array are molded within plastic prism for angular modulation based SPR signal detection. Due to mass production of these systems, 
the cost is reduced to USD about 50 . To create a multichannel SPR system [15], multiple SPREETA systems are connected in series, and the flow channel is introduced to stream the test solutions. The outputs from all the system are sent to personal computer (PC) via serial interface for signal processing. In another configuration [16], a multichannel SPR system is developed by using a prism based approach. The two-channel SPR system is demonstrated by introducing two flow channels on a single SPR chip. One channel is used as a reference and the other for sensing. Towards the signal detection, a complementary metal oxide semiconductor (CMOS) monochromatic digital sensor is used instead of a CCD array to reduce the cost of the system. The reported cost of the developed system is approximately $600 \$$. Towards cost reduction in readout systems, webcam based spectrometers are developed. The low-cost grating and webcam based spectrometer is demonstrated [17] for exposing kids to optics. Here a holographic grating with 1800 lines $/ \mathrm{mm}$ is used for light diffraction which is then focused on camera detector by using a focal lens. The lens focuses onto the slit which is developed by means of direct laser writing. The developed single channel spectrometer can be connected to laptops or tablets for signal processing. In another instance, a spectrometer is developed in the smartphone platform by using its camera [18]. Various approaches are discussed here, involving optical components such as collimators, focusing lens, mirrors, and diffraction grating. The incident angle is optimized to ensure that the diffracted light is perpendicular to the smartphone camera. The reported webcam based spectrometers are developed for single-channel application, which includes supportive optical equipments such as lens and collimators.

An optical fiber based SPR system is demonstrated by utilizing intensity modulation detection by a webcam [9]. The change in the intensity level due to the change of the surrounding $R I$ at the output of the optical fiber is captured by a webcam. Another intensity modulation based multichannel SPR system is presented by using nine optical fibers arranged in a square pattern [19]. A light emitting diode (LED) plane light source is used to couple light into the fibers. At the receiver, a lens is used to converge and focus the light coming out of the fibers onto a CMOS sensor in the cordless camera. One channel is used for reference, two channels are used for control, and six channels are used for sensing application. All six channels can be functionalized and can be used for the detection of different elements simultaneously.

In many of the presented low-cost SPR systems, intensity modulation is used with either prism or fiber configurations. CCD array [14], CMOS sensors [16] or webcam [9] are used for signal acquisition. Cost reduction up to lower than $600 \$$ is achieved by employing the inexpensive fabrication process.

In this work, a compact multichannel SPR optical transducer is presented by utilizing Bangkok University-SPR configuration [10]. Resonance dip is detected through wavelength scan. To keep the cost of the presented system low, an in-house spectrometer is developed incorporating a transmission diffraction grating and a commercially available webcam. The lens available within the webcam is utilized in the design of spectrometer. The dimension of the CCD array and the lens available in the webcam is considered vital during the design of spectrometer integrated with the SPR system. The system's performance is tested with difference RIs solutions. Due to the size of the LED sources and the limitation of the web-cam field of view as well as the imaging distance, achieving multi-channel system requires a separation between the SPR chip and the spectrometer. Using two plastic optical fibers, a two-channel SPR system is demonstrated. In both designs, the mechanical alignment for the components is provided by using the low-cost 3D printing technology, which makes the system more cost effective.

The manuscript is bifurcated into two sections. The conceptual design approach towards the development of a low-cost SPR based optical transducer, integrated with low-cost spectrometer is 
described in Section 2.1. And Section 2.2 details the design concept for multichannel SPR system with externally connected low-cost multispectral spectrometer. In the results section, theoretical response that can be achieved for the design configuration is derived using scattering matrix. It is then compared with practical response of the SPR system integrated with spectrometer (Section 3.1), and with multichannel SPR system (Section 3.2).

\section{Methods}

The demonstration of a low-cost multichannel SPR system involves designing an SPR system along with a low-cost spectrometer for spectral analysis as shown in Fig. 1.

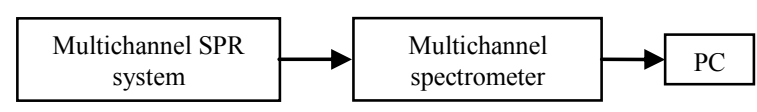

Fig. 1 Multichannel SPR system with an integrated spectrometer.

The multichannel SPR system is based on BU-SPR [10] configuration, where light is incident at the edge of the SPR chip to excite the surface plasmon wave. The signal coming out of the SPR chip is analyzed by an in-house made multichannel spectrometer. The system is developed in two steps. Firstly, a single channel system is demonstrated with an integrated spectrometer. Secondly, a two-channel system is demonstrated, where the SPR system and a multichannel spectrometer are connected by plastic optical fibers.

\subsection{Design approach for SPR transducer with integrated spectrometer}

The design approach for the SPR system integrated with a compact spectrometer using BU-SPR configuration is illustrated in Fig. 2. Here, transverse magnetic (TM) polarized light is incident at the edge of the SPR chip at an angle $\left(\theta_{a}\right)$.

The incident light is refracted at an angle $\theta_{r}$ in the glass substrate, which strikes the metal surface at an angle $\theta_{\mathrm{spr}}=90^{\circ}-\theta_{r}$. For the SPR signal in the visible spectrum and detection of liquid solutions, $\theta_{\text {spr }}$ is typically larger than the critical angle between the substrate and the cladding region. As shown in
Fig. 2, the output light is diffracted by the transmission grating, and the spectrum is recorded by the webcam.

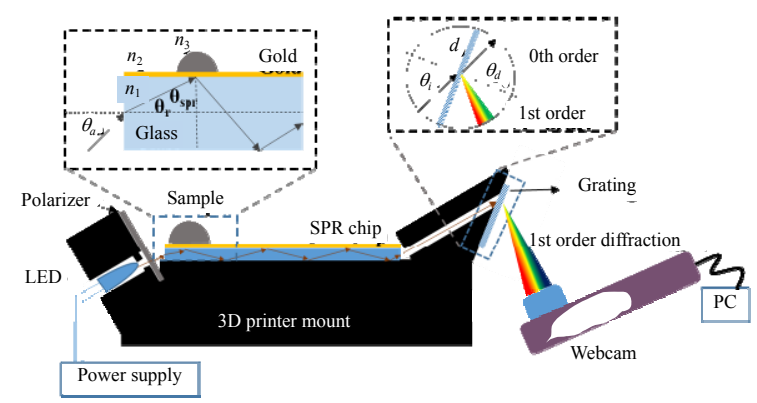

Fig. 2 Design concept for SPR system integrated with spectrometer.

In the proposed design, $\theta_{\mathrm{spr}}$ and the incident angle $\theta_{a}$ are designed to achieve an SPR dip at $600 \mathrm{~nm}$ wavelength with water $(n=1.33250)$ on the top of the sensing surface. By using the T-matrix approach, $\theta_{\mathrm{spr}}$ is calculated to be $80^{\circ}$. Further increase in $\theta_{\mathrm{spr}}$ causes the resonance wavelength to decrease. At $\theta_{\mathrm{spr}}=88^{\circ}$, the resonance wavelength reaches $590 \mathrm{~nm}$. For practical reason, $\theta_{\mathrm{spr}}$ is set to be $80^{\circ}$, hence $\theta_{r}$ is $10^{\circ}$. Based on Snell's law, $\theta_{a}$ equals $15^{\circ}$. This is the same angle at which the light exits the SPR chip.

The homemade spectrometer is composed of a transmission diffraction grating and a webcam. The light is incident on the grating at an incident angle $\theta_{i}$, and the 1 st order diffraction is captured by the webcam. As to the diffraction of angle, $\theta_{d}$ increases as the wavelength increases as depicted in Fig.3(a).

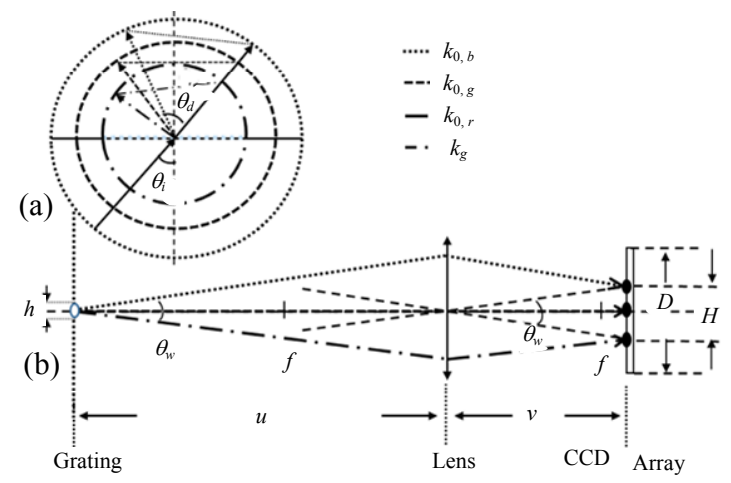

Fig. 3 Ray tracing for spectrometer design: (a) wave vector diagram used for computation of diffraction angles based on grating period and incident angle $\left(\theta_{i}\right),\left(k_{0, b}-\right.$ round dot line, $k_{0, g}$ - squared dot line, $k_{0, r}$ - long dash dot line) and (b) ray tracing demonstration of imaging system. 
In Fig. 3(a), $\theta_{i}$ and $\theta_{d}$ are the incident and diffraction angles, respectively, $k_{g}$ is the grating vector, and $k_{0, r}, k_{0, g}$, and $k_{0, b}$ are the amplitude vectors denoted by the long dash dot line $(\lambda=$ $650 \mathrm{~nm})$, squared dot line $(\lambda=550 \mathrm{~nm})$, and round dot line $(\lambda=450 \mathrm{~nm})$ respectively. The relation between $\theta_{i}$ and $\theta_{d}$ for a specific grating period $\Lambda$ and wavelength $\lambda$ is given by (1) and (2) (after substitution of vector) as

$$
\begin{gathered}
k_{d, x}=m \times k_{g}+k_{i, x} \\
k_{i, x}=\frac{2 \pi}{\lambda} \sin \theta_{i}, \quad k_{g}=\frac{2 \pi}{\Lambda}, \quad k_{d, x}=\frac{2 \pi}{\lambda} \sin \theta_{d} \\
\theta_{d}=\sin ^{-1}\left[m\left(\frac{\lambda}{\Lambda}\right)-\sin \theta_{i}\right]
\end{gathered}
$$

where $m$ is the order of diffraction, and $\theta_{d}$ is directly dependent on $\lambda$ and inversely dependent on $\Lambda$. Therefore, in this work, the angular width $\left(\theta_{w}\right)$ is defined in (3) as the difference between diffraction angles denoted by the long dashed dot line $\theta_{d, r}(650 \mathrm{~nm})$ and round dot line $\theta_{d, b}(450 \mathrm{~nm})$ :

$$
\theta_{w}=\theta_{d, r}-\theta_{d, b} \text {. }
$$

The influence of the angular width on the spectrometer design is illustrated by the ray tracing of the imaging system as shown in Fig. 3(b). Here, the object image is formed for each wavelength. The resulting image is the convolution of the object image and the light spectrum. The object distance $u$ and imaging distance $v$ are given by the space between those from object to lens and from lens to CCD array.

The relation between $v$ and $\theta_{w}$ is given as

$$
v=\left(\frac{1}{\tan \theta_{w}}\right) \times D
$$

where $D$ is the height of the CCD array. This equation is valid if the spectrum from red to blue covers the whole CCD array. Using thin lens approximation, $1 / f=1 / u+1 / v$, where $f$ is the lens focal length, the object distance $u$ and magnification $m$ can be written as

$$
\begin{gathered}
u=\frac{D \times f}{\left(\tan \theta_{w} \times f\right)-D} \\
m=\frac{\left(\tan \theta_{w} \times f\right)-D}{\tan \theta_{w} \times f} .
\end{gathered}
$$

Equations (4)-(6) indicate that $u, v$, and $m$ are influenced by $\theta_{w}$, for a specific $D$ and $f$. In our system, the USB from the webcam used has an array of $4 \mathrm{~mm}$ height $(D=5 \mathrm{~mm})$ and focal length $f=5 \mathrm{~mm}$, $D=4 \mathrm{~mm}$ ( $f$ and $D$ from the webcam). For the visible spectrum from $550 \mathrm{~nm}$ to $650 \mathrm{~nm}$, the corresponding $\theta_{w}$ (aw), $v, u$, and $m$ are plotted by varying the grating period from $430 \mathrm{~nm}$ to $1000 \mathrm{~nm}$, and it is shown in Fig. 4.

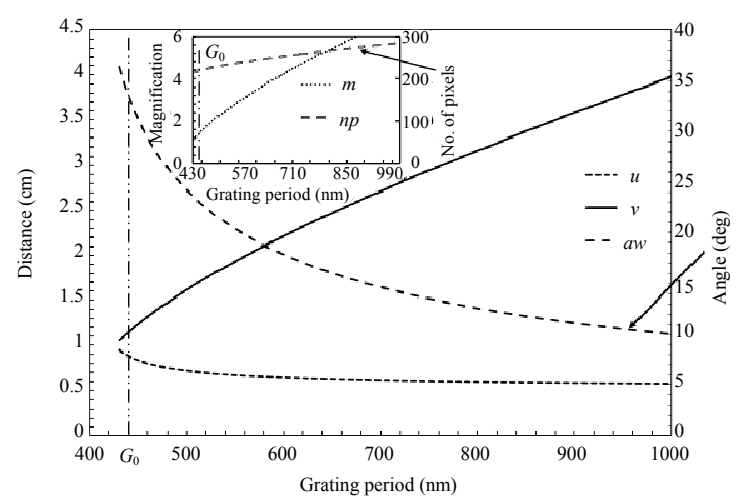

Fig. 4 Relation between $a w, u, v, m$ and number of pixels $n p$ that are covered by the spectrum $(550 \mathrm{~nm}-650 \mathrm{~nm})$, for grating period $(430 \mathrm{~nm}-1000 \mathrm{~nm})$, with the incident angle of $40^{\circ}$.

It is worth mentioning that for grating period of $430 \mathrm{~nm}$, the first order diffraction starts to present an incident angle $\theta_{d}=32^{\circ}$. Hence, in this work an incident angle of $40^{\circ}$ is selected and used in the calculations in Fig. 4. $Y$-axis in the figure is the distance in centimeter for $u$ and $v$, where in $\theta_{w}$ is angle in degrees. In the inset, plot $n p$ is the number of pixels or the spectrum length on the CCD array (here the pixel size is calculated by dividing the CCD array by 480 , corresponding to the number of pixel in a row). To compute $n p$, the height of the object $h$ is set at $1.2 \mathrm{~mm}$ (thickness of the SPR chip) and the image height $H$ is calculated from magnification (shown in the inset of Fig. 4). As the magnification increases the number of pixels covering, the spectrum increases. 
Based on these specifications, the spectrometer is designed considering a grating period $\Lambda$ of $440 \mathrm{~nm}$ ( $G_{0}$ from the Fig. 4). For $\theta_{i}=40^{\circ}$ with $\Lambda=440 \mathrm{~nm}$, the design parameters $\left(\theta_{w}, u, v, n p\right.$, and $\left.m\right)$ are shown in Table 1.

Table 1 Design parameter for spectrum to capture the 1 st order spectrum for spectral analysis.

\begin{tabular}{cccccc}
\hline Spectrum & $\theta_{w}$ & $u$ & $v$ & $n p$ & $m$ \\
\hline $550 \mathrm{~nm}-650 \mathrm{~nm}$ & $33.47^{\circ}$ & $8.8 \mathrm{~mm}$ & $11.5 \mathrm{~mm}$ & 211 & 1.3 \\
\hline
\end{tabular}

The SPR chip is fabricated by using the DC (direct current) sputtering process on the glass substrate. A thin layer of chromium of around $5 \mathrm{~nm}$ is deposited on a cleaned glass substrate, and then gold layer with thickness of $50 \mathrm{~nm}$ is deposited. The mechanical alignment is provided by using a low-cost 3D printed structure. The realized SPR system integrated with an in-house developed spectrometer is shown in Fig. 5.

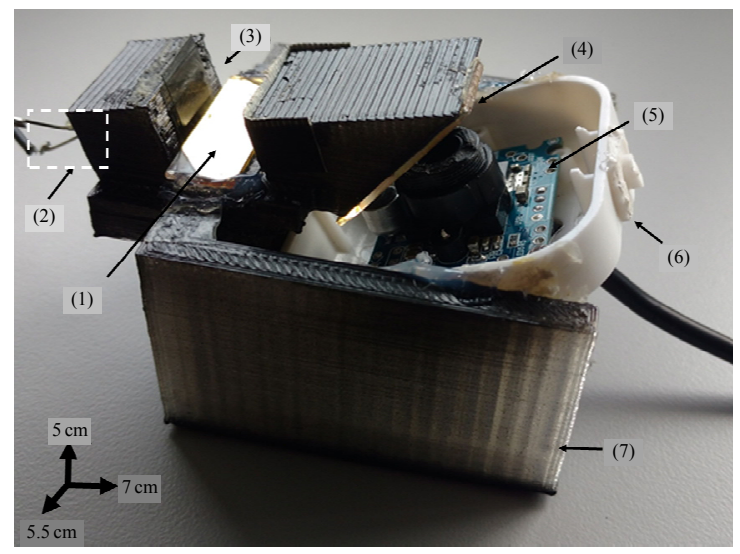

Fig. 5 Realized SPR system integrated with spectrometer along with components assembled on the 3D printed structure.

Figure 5 shows the realized SPR system with the SPR chip (1), LED (2), polarizer (3), diffraction grating (4), webcam board (5), webcam housing (6), and 3D printed structure of the system (7). An off-the-shelf LED powered by USB cable is used as the light source. A small aperture is created within the 3D structure to ensure that the incident light is only coupled to the edge of the SPR chip. A sheet polarizer is placed in between the aperture and the SPR chip, to allow TM polarization for surface plasmon excitation. The distance between the aperture and the SPR chip is set at $3 \mathrm{~mm}$. The measured width of the SPR chip is $8 \mathrm{~mm}$ and the length is $25 \mathrm{~mm}$. At the receiver section, a $2 \mathrm{~mm}$ diameter and $9 \mathrm{~mm}$ thick aperture is placed with an angle equal that of $\theta_{a}=15^{\circ}$. This is used to minimize stray light reaching the spectrometer. The light source, SPR chip, and channel are aligned in straight line.

Integrating the spectrometer, a transmission grating is sustained at $\theta_{i}=40^{\circ}$ (angle of output light is $15^{\circ}$ and tilt of the grating is $25^{\circ}$ ). The developed system has dimensions of $50 \mathrm{~mm}, 70 \mathrm{~mm}$, and $55 \mathrm{~mm}$ of length, width, and height, respectively. The acquisition is performed by using a webcam, connected to a PC for signal processing and spectrum extraction.

An additional channel can be added to the design by incorporating an additional light source and another aperture at the output. The off-shelf LEDs used in this work are $5 \mathrm{~mm}$ in diameter and the measured divergence is $12^{\circ}$ in air. Using $2 \mathrm{~mm}$ aperture, the minimum sources separation to avoid cross talk between the two sources is $4 \mathrm{~mm}$, which is less than the physical LED dimensions. Hence, the minimum separation equals that of the LED diameter. From Table 1, the field of view sets the maximum source separation to be $u \times D / f=7 \mathrm{~mm}$. Extension of the integrated design towards two channels seems feasible. However, the far off-axis placement of the two LEDs causes strong distortion in the recorded spectra. This limitation is overcome by separating the SPR chip from the multichannel spectrometer by using a pair of optical fiber cables. The design concept and realization of multichannel SPR system and multichannel spectrometer are demonstrated in Section 2.2.

\subsection{Multichannel SPR transducer}

The development of a multichannel SPR transducer follows a similar configuration to Section 2.1 , retaining $\theta_{a}, \theta_{r}$, and $\theta_{\mathrm{spr}}$. The difference is the use of two light sources. The two SPR signals are coupled with two plastic optical fibers for spectral 
analysis in a separate multichannel spectrometer as depicted in Fig. 6.

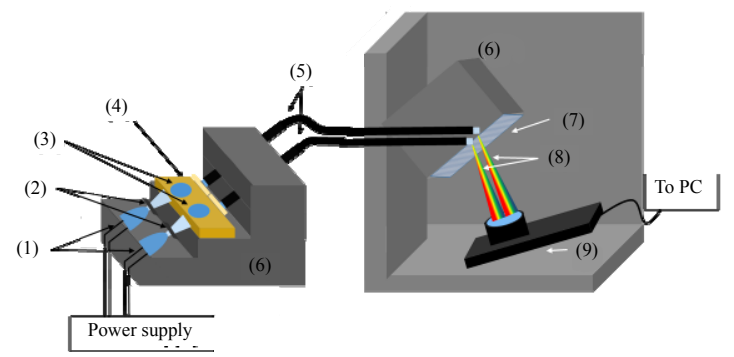

Fig. 6 Multichannel SPR system and multichannel spectrometer design.

The system comprises of two individual LED light sources (1), two polarizers (2), sample solution drops (3), and single SPR chip (4). The SPR signal is collected with two optical fibers (5) and incident at diffraction grating (7) at $\theta_{i}=50^{\circ}$. The 1 st order diffraction (8) of the two sources is captured by the webcam (9). The mechanical alignment is provided by a $3 \mathrm{D}$ printed structure (6) for both the SPR system and spectrometer. $\theta_{i}$ is increased to $50^{\circ}$ to reduce $\theta_{d}$ which reduces $\theta_{w}$, and hence the spectrum covering $450 \mathrm{~nm}$ to $650 \mathrm{~nm}$ can be captured in a single frame. Due to the change in $\theta_{i}$, the values of $\theta_{w}, v, u, m, a w$, and $n p$ are all altered. This can be determined using (2)-(6). Solving the equations for $\theta_{i}=50^{\circ}, f=5 \mathrm{~mm}$, pixel height of $120 \mu \mathrm{m}$, visible light spectrum from $450 \mathrm{~nm}$ to $650 \mathrm{~nm}$, and varying $\Lambda$ from $430 \mathrm{~nm}$ to $1000 \mathrm{~nm}$, leads to the result shown in Fig. 7.

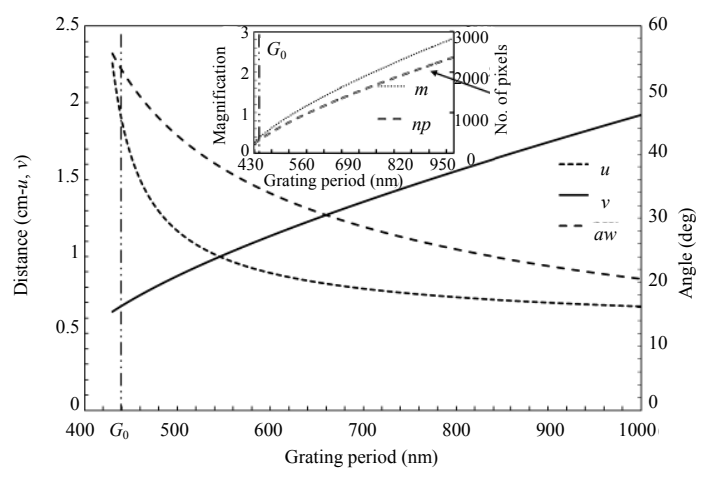

Fig. 7 Relation between angular width $a w$, object distance $u$, image distance $v$, magnification $m$ and number of pixels $n p$, for grating period from $430 \mathrm{~nm}-1000 \mathrm{~nm}$ with the incident angle of $50^{\circ}$.

From Fig. 7, the values pertaining to $\Lambda$ of $440 \mathrm{~nm}$ (marked as $G_{0}$ ) are considered for the design approach. The corresponding values for $\theta_{w}(a w), v, u$, $n p$ and $m$ are given in Table 2 .

Table 2 Design parameters for multichannel spectrometer to capture the 1st order spectrum for spectral analysis.

\begin{tabular}{cccccc}
\hline Spectrum & $\theta w$ & $u$ & $v$ & $n p$ & $m$ \\
\hline $450 \mathrm{~nm}-650 \mathrm{~nm}$ & $53.17^{\circ}$ & $18 \mathrm{~mm}$ & $6.8 \mathrm{~mm}$ & 360 & 0.36 \\
\hline
\end{tabular}

Following the achieved design parameters, the system is realized and is shown in Fig. 8. The two light sources (1) are positioned at a distance of $10 \mathrm{~mm}$ apart.

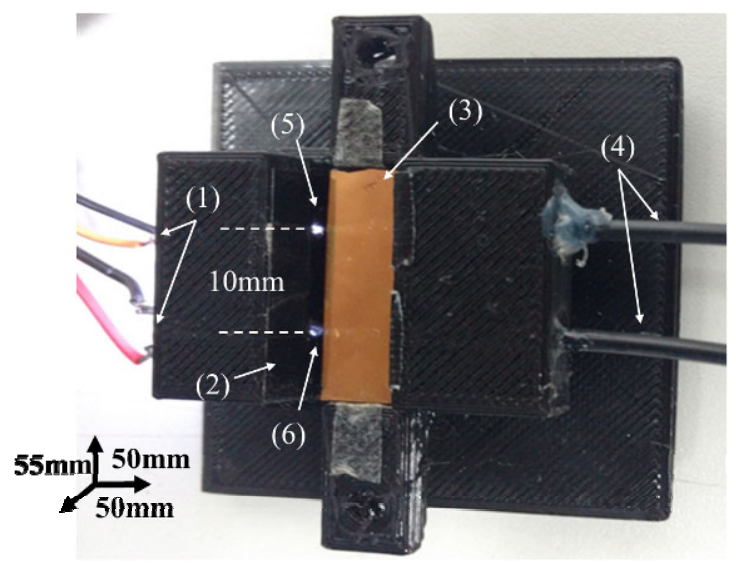

Fig. 8 Realized 3D printed multichannel SPR system with components assembled.

The diameter of the aperture at the source is $1 \mathrm{~mm}$, the distance between Channel 1 (5) and Channel 2 (6) is $10 \mathrm{~mm}$, and the distance between sources to SPR chip is set at $3 \mathrm{~mm}$. The polarizer (2) is placed in front of the aperture. The width and length of SPR chip (3) are $8 \mathrm{~mm}$ and $25 \mathrm{~mm}$, respectively. At the receiver section, two optical fiber cables (4) with core diameter of $1 \mathrm{~mm}$ are placed very close to the SPR chip to couple reflected light into optical fiber. The fibers are positioned at an angle equal to $\theta_{a}$ and are extended to connect with the spectrometer. The dimensions of the SPR system is $55 \mathrm{~mm}$ in length, $50 \mathrm{~mm}$ in width, and $50 \mathrm{~mm}$ in height, making it a compact multichannel SPR system.

The fibers, collecting the SPR signals, are connected to an external spectrometer for spectral analysis. The 3D printed structure provides angular 
alignment for the grating and fibers to control the incident angle of light. There is a $1 \mathrm{~mm}$ separation between the fibers. They are kept closer to capture fiber edges within the field of view, and two spectra can be fit in one frame. The grating is placed at the edge of the fibers, and the first order diffracted light is captured by using the webcam. The design approach of the spectrometer follows the parameters mentioned in Table 2. Image processing is done to extract the spectral details from the captured spectrum. The change in intensity corresponding to the dip is recorded.

The system design is theoretically analyzed to determine the sensitivity slope. The developed systems are tested with solutions of different RIs. The theoretical response is compared with experimental results.

The test samples of different RIs are prepared by using glycerol solution of different concentrations. The $R I$ of the samples prepared is measured by using a digital refractometer (Rx-7000, ATAGO USA).

\section{Experimental results and discussion}

The theoretical analysis for the SPR system is carried by deriving the scattering matrix (S-matrix) $[20,21]$ for single layer (gold) of finite thickness $(50 \mathrm{~nm})$ and infinite substrate and cladding thicknesses. In solving S-matrix, a TM polarized plane wave is considered to propagate from the substrate to incident $\left(\theta_{\text {spr }}\right)$ at the substrate-metal interface. The amplitude of incoming waves, propagating in the metal and reflecting back into the substrate, is related with the scattering matrix. Similarly, at the metal cladding interface, light is transmitted into cladding and is also reflected back into the metal layer. The amplitude of reflected light into the substrate is analyzed by changing the $R I$ of cladding. For the substrate of $R I 1.5$, the cladding $R I$ is varied from 1.33250 to 1.33951 . The theoretical response for $\theta_{\mathrm{spr}}=80^{\circ}$ is shown in Fig. 9 .

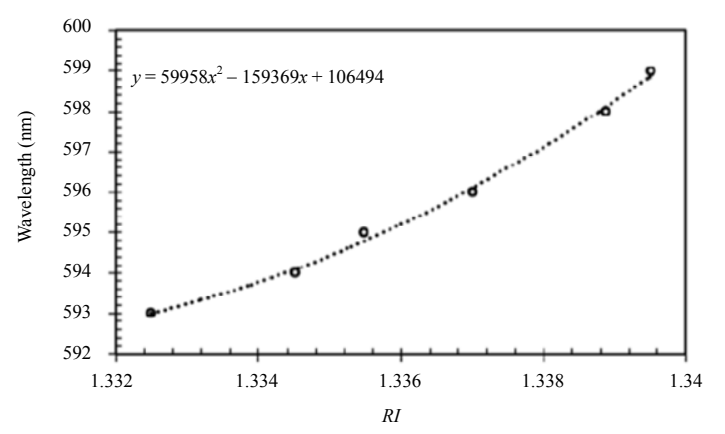

Fig. 9 Theoretical response for the different cladding $R I s$ for the followed design approach.

\subsection{SPR transducer with integrated spectrometer}

Using the design approach (Section 2.1), the spectrum of SPR signal is captured by the webcam. The captured frame is cropped and shown in Fig. 10(a). The brightness of the image is enhanced by $50 \%$ to improve the visibility.

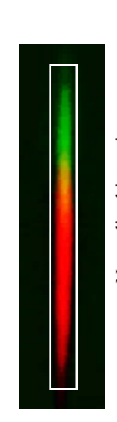

(a)

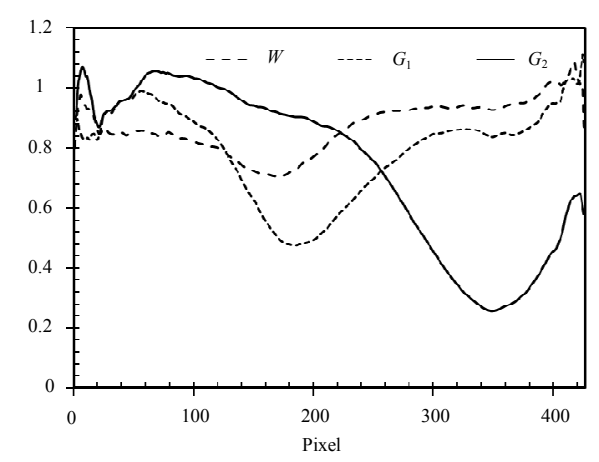

(b)
Fig. 10 Spectrum captured by the developed system: (a) the cropped image acquired by the webcam of a spectrum covering green and red color, and also marked with region of interest for image processing and (b) intensity variation for water $(W=$ $1.33250)$ and glycerol solution $\left(G_{1}=1.33547\right.$ and $\left.G_{2}=1.33639\right)$.

The cropped frame covers the color from green to red from the visible spectrum. The removal of the UV (ultra violet) filter from the webcam allows UV light to reach the CCD array. This increases the spectrum length to 236 pixels and is higher by 25 pixels from theoretical value (211). Along with filter removal, the possible variation in $u$ and $v$, caused during the arrangement in the $3 \mathrm{D}$ printed structure, results in longer spectral occupancy. The process involves image processing to extract spectral details from the selected region in order to find the dip position, which is explained in Fig. 11. 


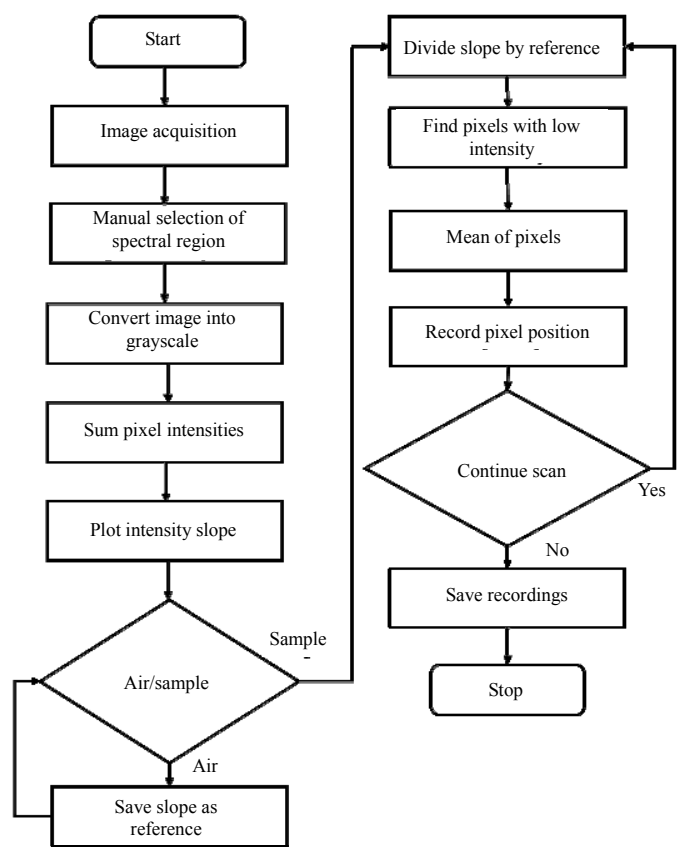

Fig. 11 Image processing involved in spectrum analysis for extraction of spectral data and finding the dip position.

Starting with the image acquisition from a webcam, the region covering the spectrum is manually selected as shown in Fig. 10(a). The selected region is converted into gray scale, and the intensity of the pixels is summed horizontally. The total intensity response results in a slope of intensity versus pixel position. Based on the cladding region, the slope response is analyzed. A two-step process is required. Firstly, the cladding is air, the slope response is recorded as reference and then the cladding is sample, and its recorded value is divided by the reference to find the dip position. The response curve for water and glycerol solutions $G_{1}$ and $G_{2}$ is shown in Fig. 10(b).

To find the dip from the response curve, a threshold is set to track the number of pixels (20), with low intensity, and the mean of the pixel position is recorded. The process is repeated for 50 reading with time interval of $1 \mathrm{~s}$, and also with different RIs solutions. The response of SPR system integrated with spectrometer is shown in the inset of Fig. 12.

The system response with time is recorded starting with water on the top of the sensing surface, and after 50 readings the sample is cleaned, and the second sample $G_{1}$ is placed. The cleaning process is noticeable as noise between the sample responses. The process is repeated with the solutions $G_{2}, G_{3}$, and $W$ again, hence the recorded response of the system is shown in the inset of Fig. 12. The system is tested several times and observed that the system response is repeatable and stable. Figure 12 shows the dip position for every solution and its standard deviation, demonstrating a nonlinear response. A 2nd order polynomial curve is fitted to obtain the polynomial equation. Using the equation and the standard deviation, the minimum sensitivity achieved by the system at lower $R I(W=1.33250)$ is $4.95 \times 10^{-4} \mathrm{RIU}$, and the maximum at higher $R I\left(G_{3}=\right.$ 1.33887 ) is $2.37 \times 10^{-4} \mathrm{RIU}$.

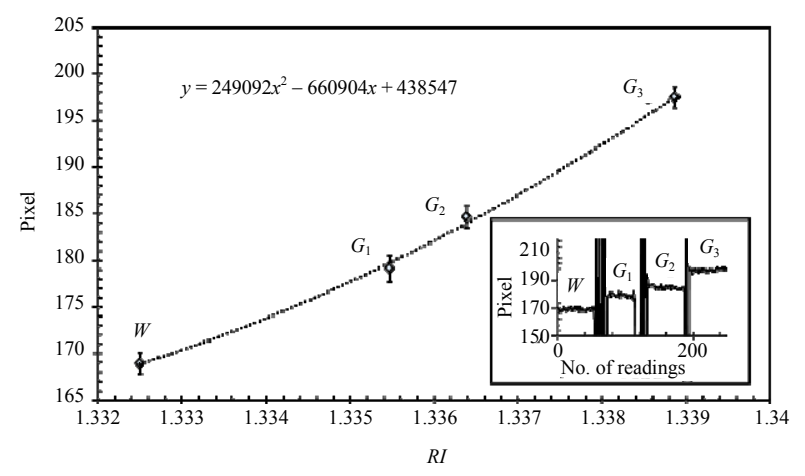

Fig. 12 Plot of dip position with respect to RI. Inset, system response for solutions: water $(W=1.33250)$, and glycerol of different $R I s\left(G_{1}=1.33547, G_{2}=1.33639\right.$, and $\left.G_{3}=1.33887\right)$.

\subsection{Multichannel SPR system with multichannel spectrometer}

In the multichannel SPR system, two spectra covering blue $(450 \mathrm{~nm})$ to red $(650 \mathrm{~nm})$ colors are captured by the spectrometer as shown in Fig. 13(a).

The fiber edge is placed at proximity to the grating, therefore the aperture diameter is similar to the diameter of the fiber core, which is $1.2 \mathrm{~mm}$. The spectra captured are the convolution of three images (blue, green, and red) and hence the recorded width dimension $1.8 \mathrm{~mm}$. The spectrum lies within the field of view of the webcam with short focal length $(5 \mathrm{~mm})$ and diameter $(2.5 \mathrm{~mm})$, and hence the 
spectrum of Channel 2 is tilted. The webcam is aligned to capture the spectrum of Channel 1 in straight and Channel 2 spectrum undergoes aberration and appears to be tilted.

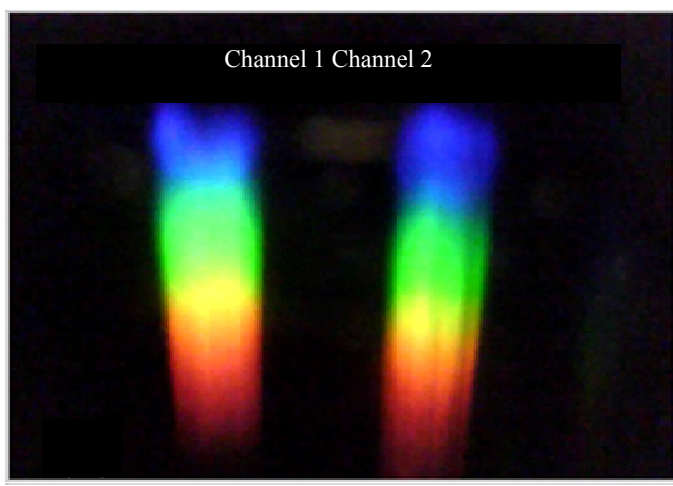

(a)

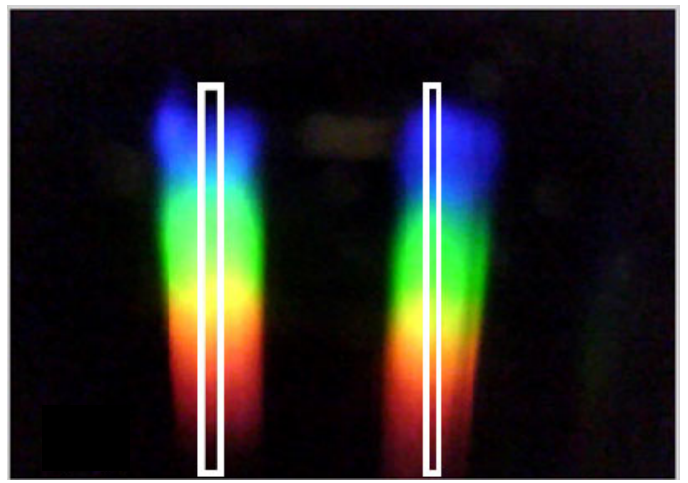

(b)

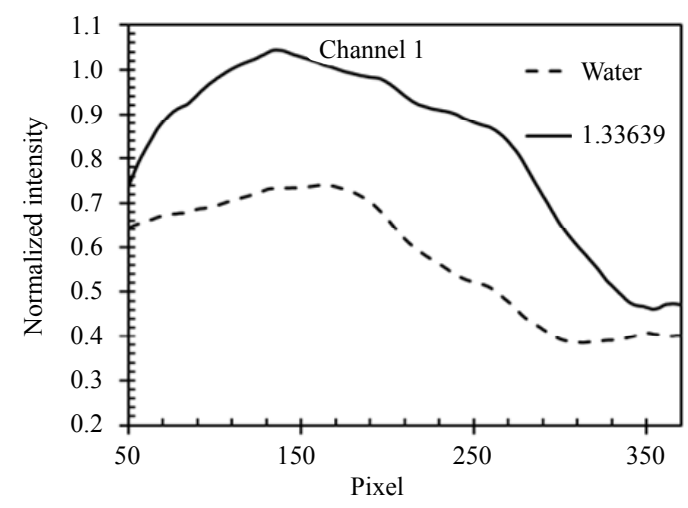

(c)

Fig. 13 Spectra captured by multi-channel spectrometer: (a) spectrum of Channels 1 and 2 at a distance apart $(1.6 \mathrm{~mm})$, (b) selection of area of interest for spectral analysis, and (c) change in intensity level for different RIs from Channel 1.

In order to test the system, image processing is performed for the spectra using two different approaches. At first, the image is processed using the algorithm detailed in Fig. 11. It follows manual selection of the region of interest as shown in Fig. 13(b) and testing with solutions of different RIs. The response of Channel 1 for water (1.33250) and solution of RI 1.33639 is shown in Fig. 13(c). Following this approach, Channels 1 and 2 are tested to determine the sensitivity, and the dip positions are recorded as shown in Fig. 14.

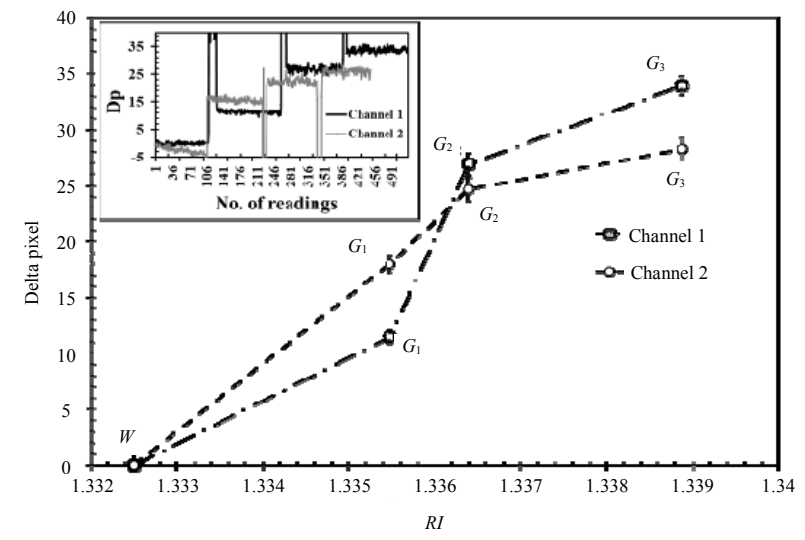

Fig. 14 Response of Channels 1 and 2 for different RI solutions, plotted with respect to delta pixel. The inset shows the recording of system response with different solutions.

Both the channels are tested with $W, G_{1}, G_{2}$, and $G_{3}$, and their responses are recorded for 105 times with time interval of $1 \mathrm{~s}$. The response of Channels 1 and 2 can be seen in dark and light colors, respectively, in the inset of Fig. 14. The testing starts with water solution, and then sensing surface is cleaned before the sample $G_{1}$ is placed. This process is repeated for $G_{2}$ and $G_{3}$, while the recording is still on. The noise recorded during the cleaning process is observed as very high and low values. The deviation of dip position, caused due to the change in $R I$, is recorded as delta pixel, considering the position at which the dip occurs for water on sensing surface as the starting pixel. The plot of pixel position with respect to RIs for both Channels 1 and 2, along with its standard deviation, is shown in Fig. 14. The sensitivity achieved at lower $R I(W)$ for Channels 1 and 2 are in the order of $2.02 \times 10^{-4}$ RIU and $1.27 \times 10^{-4}$ RIU, respectively. The sensitivity achieved at higher $R I\left(G_{3}\right)$ for Channels 1 and 2 are 
in the order of $1.08 \times 10^{-4} \mathrm{RIU}$ and $1.72 \times 10^{-4} \mathrm{RIU}$, respectively. Following the above image processing approach, only one spectrum is manually selected and analyzed. Towards analyzing both spectra simultaneously and testing the channels, another approach of image processing is followed, which is explained in Fig. 15.

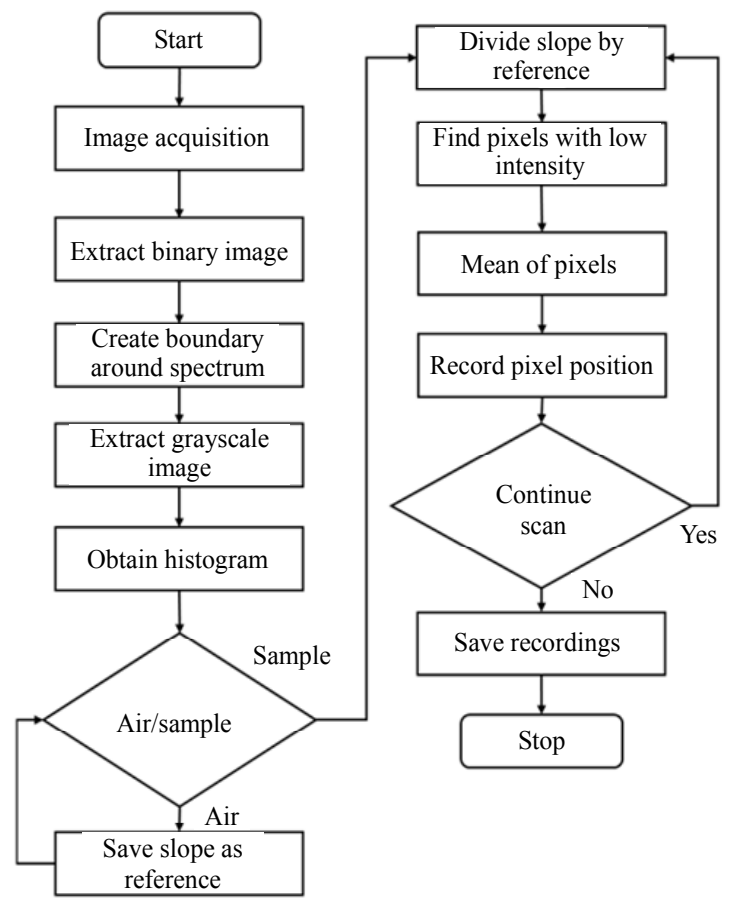

Fig. 15 Second approach of image processing, involved in automatic selection of spectra, and its analysis for finding the dip position.

Here, the captured frame is at first converted into binary image as shown in Fig. 16(b). The pixels covering the spectrum are shown in white color, which allows in automatic detection of the spectral region. A rectangular boundary is generated around the pixels covering the spectrum, and a grayscale image of the selected region is extracted from the original image. The histogram of grayscale image provides the intensity response slope with respect to pixel position. Following the process, the steps involved in collecting the reference and channel response for different solutions are similar to steps explained in Fig. 11.

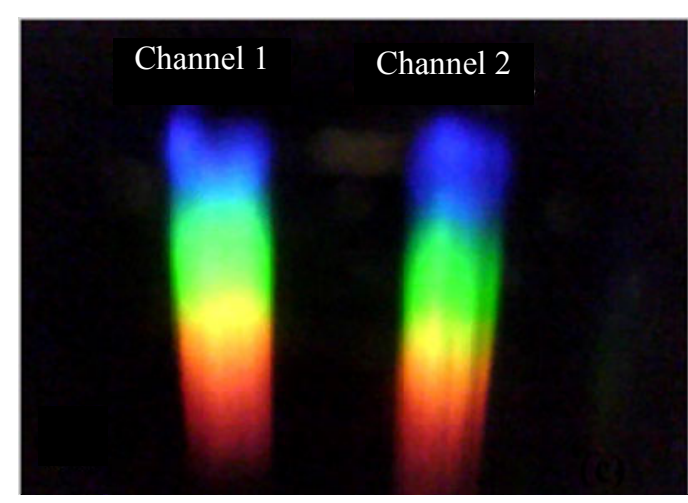

(a)

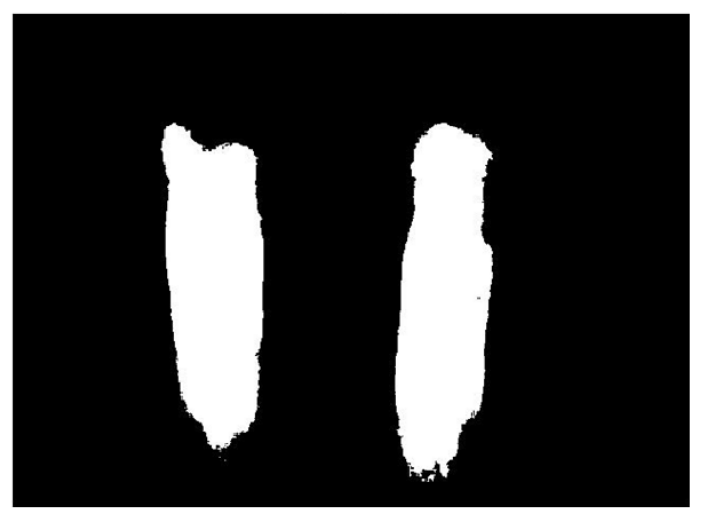

(b)

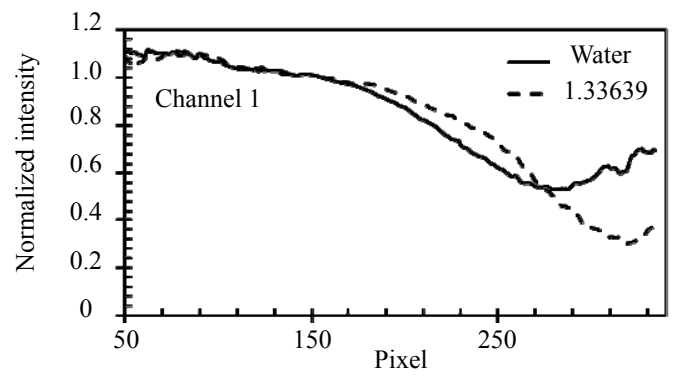

(c)

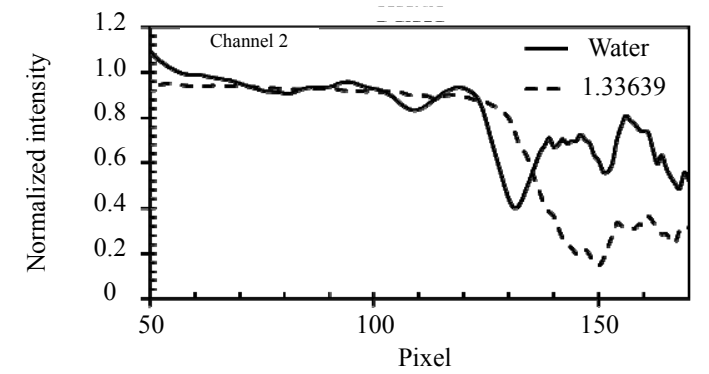

(d)

Fig. 16 Data extraction from two spectra: (a) spectrum captured by multi-channel spectrometer, (b) the binary image of the frame where the spectrum is highlighted by white pixels, indicating the region of interest, (c) intensity response of Channel 1 for water, and solution of RI 1.33639, and (d) intensity response of Channel 2 for water and solution of $R I$ 1.33639 . 
Following the second approach of image processing, Channels 1 and 2 are tested with water, and solution of $R I 1.33639$, and their intensity change response is shown in Figs. 16(c) and 16(d). While testing both the channels simultaneously, Channel 2 is considered as reference by dropping water on the sensing surface throughout the experiment, and Channel 1 is tested with $W, G_{2}$, and $G_{3}$ solutions. The system responses from both channels are shown in the inset of Fig. 17.

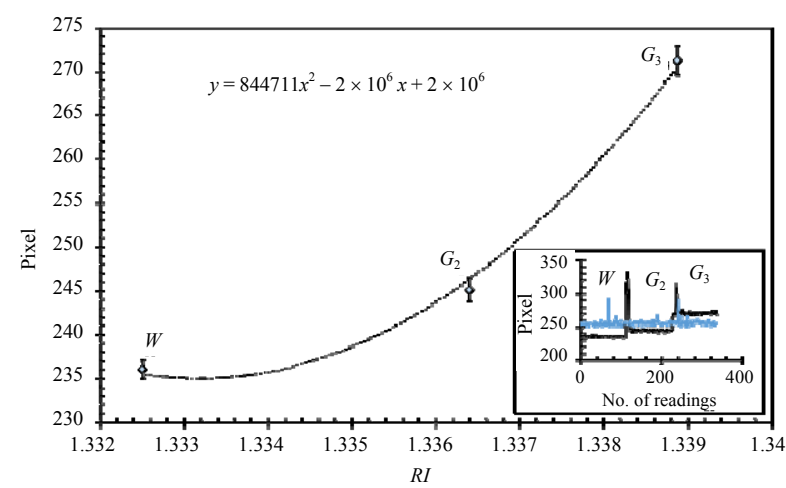

Fig. 17 Plot of dip positions with respect to RI for Channel 1. The inset shows the recording of response from Channel 1 (dark) and Channel 2 (light).

Response of Channel 2 (light) with water as sample is considered as reference, and response from Channel 1 is recorded with solutions $G_{2}$ and $G_{3}$. The pixel position with respect to $R I$ measured for Channel 1 is shown in Fig. 17. The sensitivity recorded for Channel 1 is in the order of $1.2 \times 10^{-4} \mathrm{RIU}$. The pixels position for $W$ sample is different for both the channels, because both the spectra are not identical and do not have similar length.

As explained earlier in the spectrometer design approach, the object size is similar to the one of the fiber core diameter $(1.2 \mathrm{~mm})$, without any aperture. Towards study of influence of aperture in the designed spectrometer and with followed peak detection scheme, a small aperture of $200 \mu \mathrm{m}$ is placed in front of the grating, and Channel 1 is tested with solutions of different RIs. The responses of the system is recorded and compared with the responses recorded without aperture and is shown in Fig. 18.

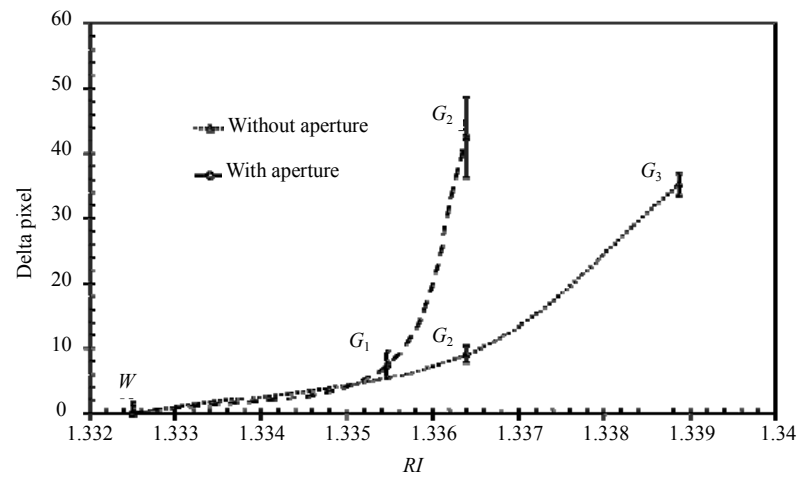

Fig. 18 Response of Channel 1, with and without aperture, following the second approach of image processing.

For the system with aperture, Channel 1 is tested with water, $G_{1}$, and $G_{2}$ solutions. Due to the presence of the aperture, the intensity of light drops near higher wavelengths, and hence the solution $G_{2}$ demonstrates higher standard deviation.

The sensitivity measured with the presence of aperture is $1.33 \times 10^{-4} \mathrm{RIU}$ at higher $R I$, which is lower than that without aperture $\left(1.23 \times 10^{-4} \mathrm{RIU}\right)$. Therefore, no significant change in sensitivity is observed, wherein the response is nonlinear.

The developed low-cost SPR system integrated with spectrometer and multichannel SPR system with multichannel spectrometer has demonstrated sensitivity in the order of $10^{-4} \mathrm{RIU}$ and is close to the theoretically achievable sensitivity. The low-cost spectrometers are developed by using an optical diffraction grating and a webcam and are used for both single channel and multichannel. The multichannel spectrometer can also be used for detection of two different targeted materials. The theoretical design parameters provided for the design of two-channel spectrometer can be used for easy development for various applications. Depending upon the webcam specifications, the optimal conditions for alignments of components can be calculated in developing a spectrometer. The application of the low-cost 3D printing technology brings down the cost involved in developing the system. Therefore, the cost involved in developing 
the SPR system with integrated spectrometer approximates $35 \$$. With the addition of extra light source and optical fibers to interface SPR system with spectrometer, it keeps the cost of overall system lower than $40 \$$. The cost of the systems can be further reduced by mass production. The developed multichannel SPR system can be used for detection of two individual parameters in a sample way, simultaneously, by functionalizing the surface.

The developed systems using the low-cost 3D printing technology allow researchers to develop a low-cost SPR system for various sensing applications. The resolution of the spectrometer can be varied upon selection of webcams for the design purpose. With the sensitivity achieved, the sensor can be used for the detection of chemicals such as presence of mercury [22, 23] in water, where $100 \mu \mathrm{M}$ of mercury results in change of refractive index in the order of $10^{-4}$ RIU. Therefore, the chemicals as low as $100 \mu \mathrm{M}$ can be detected with the developed system. The sensitivity of the system can be further improved by dropping high $R I$ solution only at the position where the SPR phenomena firstly occurs. Also by introducing a flow channel on the sensing surface, the area of interaction can be controlled.

According to the survey, chemicals, such as fluorine and zinc [24] whose presence is in water up to a few $\mathrm{mg}$, are detected in various water samples in many countries across the world, which is health hazardous. The developed system can be used for the detection of such chemicals and their concentration level can be determined. It also provides threshold based warnings when employed in remote locations for early detection and warning of chemical imbalance in biological samples. It is worth mentioning that all the experiments are performed in ambient environment conditions, and hence it is well suited for field applications.

The developed low-cost multichannel spectrometer using a low-cost technology can also be used for various other applications such as astrophysical observations [25], astrophotonics [26], remote chemical detection [27], and sensor application based on guided mode resonance (GMR) [28].

\section{Conclusions}

The demonstration of a low-cost, portable, and compact SPR based optical transducer is realized following the BU-SPR approach. The plasmon excitation angle is firstly obtained by deriving the scattering matrix, and based on the angle, the SPR system is developed. At first, a single-channel SPR transducer is developed and integrated with a webcam and grating based in-house developed low-cost spectrometer.

The multichannel SPR system is also realized by using the BU-SPR approach by adding an extra light source and using optical fibers for interface SPR signal with separated multichannel spectrometer for spectra analysis. Both channels are tested separately to determine the sensitivity of each channel. Following the manual selection of spectrum for image processing, the channels are tested simultaneously by using the automatic spectra selection approach. While simultaneously testing the channels, Channel 2 is considered as reference and Channel 1 is used for sensing. Image processing is performed for spectral analysis and to dip position detection. The achieved sensitivity response closely follows the similar response as theoretical data.

The physical alignment involved in developing both the systems is provided by using low-cost 3D printed structures. Both the developed systems are tested with water and glycerol solutions of different concentrations. The sensitivity of the system is measured to be in the order of $10^{-4}$. The cost of SPR system integrated with spectrometer is about 35\$, and multichannel SPR system with spectrometer is lower than $45 \$$. The cost of the systems can be further reduced by mass production. 
Open Access This article is distributed under the terms of the Creative Commons Attribution 4.0 International License (http://creativecommons.org/licenses/by/4.0/), which permits unrestricted use, distribution, and reproduction in any medium, provided you give appropriate credit to the original author(s) and the source, provide a link to the Creative Commons license, and indicate if changes were made.

\section{References}

[1] M. G. Manera and R. Rella, "Improved gas sensing performances in SPR sensors by transducers activation," Sensors and Actuators B: Chemical, 2013, 179(4): 175-186.

[2] R. Galatus, B. Feier, C. Cristea, N. Cennamo, and L. Zeni, "SPR based hybrid electro-optic biosensor for $\beta$-lactam antibiotics determination in water," SPIE, 2017, 10405: 104050C-1-104050C-6.

[3] F. Geiss, S. Fossati, I. Khan, N. G. Quilis, W. Knoll, and J. Dostalek, "UV-SPR biosensor for biomolecular interaction studies," SPIE, 2017, 10231: 1023107-1-1023107-8.

[4] H. H. Kyaw, S. Boonruang, W. S. Mohammed, and J. Dutta, "Design of electric-field assisted surface plasmon resonance system for the detection of heavy metal ions in water," AIP Advances, 2015, 5(10): 246-253.

[5] Y. H. Choi, G. Y. Lee, H. Ko, Y. W. Chang, M. J. Kang, and J. C. Pyun, "Development of SPR biosensor for the detection of human hepatitis $\mathrm{B}$ virus using plasma-treated parylene-N film," Biosensors and Bioelectronics, 2014, 56(56): 286-294.

[6]X. L. Zhang, Y. Liu, T. Fan, N. Hu, Z. Yang, X. Chen, et al., "Design and performance of a portable and multichannel SPR device," Sensors, 2017, 17(6): 1435-1-1435-7.

[7] S. Maegawa, J. Yamaguchi, F. Itoigawa, and T. Nakamura, "Discussion on surface plasmon resonance technique in the Otto configuration for measurement of lubricant film thickness," Tribology Letters, 2016, 62(2): 1-14.

[8] C. Thirstrup, W. Zong, M. Borre, H. Neff, H. C. Pedersen, and G. Holzhueter, "Diffractive optical coupling element for surface plasmon resonance sensors," Sensors and Actuators B: Chemical, 2004, 100(3): 298-308.

[9] Q. Liu, Y. Liu, S. M. Chen, F. Wang, and W. Peng, "A low-cost and portable dual-channel fiber optic surface plasmon resonance system," Sensors, 2017, 17(12): 2797-1-2797-8.

[10] M. Somarapalli, K. Koul, R. Lahon, S. Boonruang, and W. S. Mohammed, "Demonstration of low-cost and compact SPR optical transducer through edge light coupling," Micro \& Nano Letters, 2017, 12(9): 643-646.
[11] S. Nizamov, V. Scherbahn, and V. M. Mirsky, "Self-referencing SPR-sensor based on integral measurements of light intensity reflected by arbitrarily distributed sensing and referencing spots," Sensors and Actuators B: Chemical, 2015, 207: 740-747.

[12] X. L. Zhou, K. Chen, L. Li, W. Peng, and Q. X. Yu, "Angle modulated surface plasmon resonance spectrometer for refractive index sensing with enhanced detection resolution," Optics Communications, 2017, 382: 610-614.

[13] H. Zhang, D. Q. Song, S. Gao, H. Q. Zhang, J. Zhang, and Y. Sun, "Enhanced wavelength modulation SPR biosensor based on gold nanorods for immunoglobulin detection," Talanta, 2013, 115(115): 857-862.

[14] T. M. Chinowsky, J. G. Quinn, D. U. Bartholomew, R. Kaiser, and J. L. Elkind, "Performance of the Spreeta 2000 integrated surface plasmon resonance affinity sensor," Sensors and Actuators B: Chemical, 2003, 91(1): 266-274.

[15] G. Neuert, S. Kufer, M. Benoit, and H. E. Gaub, "Modular multichannel surface plasmon spectrometer," Review of Scientific Instruments, 2005, 76(5): 054303-1-054303-4.

[16] S. Rampazzi, G. Danese, F. Leporati, and F. Marabelli, "A localized surface plasmon resonance-based portable instrument for quick on-site biomolecular detection," IEEE Transactions on Instrumentation and Measurement, 2016, 65(2): 317-327.

[17] I. Khodadad, N. Abedzadeh, V. Lakshminarayan, and S. S. Saini, "Low cost spectrometer and learning applications for exposing kids to optics," SPIE, 2015, 9793: 97932W-1-97932W-5.

[18] A. J. S. McGonigle, T. C. Wilkes, T. D. Pering, J. R. Willmott, J. M. Cook, F. M. Mims, et al., "Smartphone spectrometers," Sensors, 2018, 18(1): 223-1-223-15.

[19] Y. Liu, S. Chen, Q. Liu, J. F. Masson, and W. Peng, "Compact multi-channel surface plasmon resonance sensor for real-time multi-analyte biosensing," Optics Express, 2015, 23(16): 20540-20548.

[20] D. Whittaker and I. Culshaw, "Scattering-matrix treatment of patterned multilayer photonic structures," Physical Review B, 1999, 60(4): 2610-2618.

[21] R. C. Hall, R. Mittra, and K. M. Mitzner, "Analysis of multilayered periodic structures using generalized scattering matrix theory," IEEE Transactions on Antennas and Propagation, 1988, 36(4): 511-517.

[22] S. Chah, J. Yi, and R. N. Zare, "Surface plasmon resonance analysis of aqueous mercuric ions," Sensors and Actuators B: Chemical, 2004, 99(2): 216-222.

[23] P. Zhang, Y. P. Chen, W. Wang, Y. Shen, and J. S. Guo, "Surface plasmon resonance for water pollutant 
detection and water process analysis," Trends in Analytical Chemistry, 2016, 85: 153-165.

[24] L. Goldman and A. I. Schafer, Goldman's cecil medicine E-book. Oxford, UK: Elsevier Health Sciences, 2011: 1-2704.

[25] A. S. Shcherbakov, A. O. Arellanes, and V. Chavushyan, "Optical spectrometer with acousto-optical dynamic grating for guillermo haro astrophysical observatory," International Journal of Astronomy \& Astroph, 2013, 3(4): 376-384.
[26] N. Blind, E. L. Coarer, P. Kern, and S. Gousset, "Spectrographs for astrophotonics," Optics Express, 2017, 25(22): 27341-27369.

[27] E. C. Cull, M. E. Gehm, S. T. McCain, B. D. Guenther, and D. J. Brady, "Multimodal optical spectrometers for remote chemical detection," SPIE, 2005, 5778: 376-383.

[28] W. K. Kuo and C. J. Hsu, "Two-dimensional grating guided-mode resonance tunable filter," Optics Express, 2017, 25(24): 29642-29649. 